\title{
GROTHENDIECK GROUPS AND DIVISOR GROUPS ${ }^{1}$
}

\author{
ROBERT M. FOSSUM
}

0. Introduction. Before stating the results in this note, it is necessary to introduce some notation. $A$ is a noetherian integral domain which is in tegrally closed in its quotient field $K$. $\Sigma$ is a central simple finite-dimensional $K$-algebra, $D$ is a central division $K$-algebra and $V$ is a finitely generated right $D$ vector space such that $\Sigma=\operatorname{Hom}_{D}(V, V)$ (so also $D=\operatorname{Hom}_{\Sigma}(V, V)$ ).

Let $\Lambda$ be an $A$-order in $\Sigma$. $\mathscr{T}(\Lambda)$ denotes the category of left finitely generated $\Lambda$-modules, $\mathfrak{J}(\Lambda)$ the Serre subcategory of $\mathfrak{T}(\Lambda)$ consisting of $A$-torsion left $\Lambda$-modules. $\rho(\Lambda)$ is the Serre subcategory of $J(\Lambda)$ consisting of the pseudo-nul left $\Lambda$-modules, where a pseudonul module $M$ is one for which $M_{\mathfrak{p}}=A_{\mathfrak{p}} \otimes_{A} M=0$ for all prime ideals $\mathfrak{p}$ of $A$ of height at most one. The category $\mathfrak{T} / \mathcal{P}(\Lambda)$ is formed by taking as objects the objects of $\mathscr{T}(\Lambda)$ and for $M, N$ in $\mathscr{T}(\Lambda)$, defining $\operatorname{Hom}_{\mathfrak{T} / \mathcal{}}(M, N)$ to be the direct limit of $\operatorname{Hom}_{\mathfrak{T}}\left(M^{\prime}, N^{\prime}\right)$ taken over those $M^{\prime}$ and $N^{\prime}$ such that $M / M^{\prime}$ is in $\odot$ and $N^{\prime}=N / N^{\prime \prime}$ with $N^{\prime \prime}$ in $\odot . J / P(\Lambda)$ is formed in a similar fashion. The first result may now be stated as follows:

Theorem 1. Let $A, \Sigma, D$ be as above. Let $\Lambda_{1}$ and $\Lambda_{2}$ be maximal orders in $\Sigma$, and $\Gamma$ a maximal order in $D$. Then there are functors

$$
\begin{aligned}
& F\left(\Lambda_{1}, \Lambda_{2}\right): \mathfrak{T}\left(\Lambda_{1}\right) \rightarrow \mathscr{T}\left(\Lambda_{2}\right), \\
& G\left(\Lambda_{2}, \Gamma\right): \operatorname{Tr}\left(\Lambda_{2}\right) \rightarrow \operatorname{Tr}(\Gamma),
\end{aligned}
$$

which induce isomorphisms of the categories

$$
\begin{aligned}
\mathfrak{T} / \mathrm{P}\left(\Lambda_{1}\right) & \rightarrow \mathfrak{T} / \odot\left(\Lambda_{2}\right) \rightarrow \mathfrak{T} / \odot(\Gamma), \\
J / P\left(\Lambda_{1}\right) & \rightarrow J / P\left(\Lambda_{2}\right) \rightarrow J / \odot(\Gamma) .
\end{aligned}
$$

If $\mathfrak{C}$ is an abelian category, $K^{0}(\mathcal{C})$ denotes the Grothendieck group of $\mathcal{C}$. It can be defined as follows: For each $C$ in $\mathcal{C}$ there is an $f(C)$ in $K^{0}(\mathcal{e})$, an abelian group, such that if $0 \rightarrow C^{\prime} \rightarrow C \rightarrow C^{\prime \prime} \rightarrow 0$ is an exact sequence in $\mathfrak{e}$, then $f(C)=f\left(C^{\prime}\right)+f\left(C^{\prime \prime}\right)$. Furthermore, if $G$ is any abelian group and for each $\mathcal{C}$ in $C$ there is a $g(C)$ in $G$ such that $g(C)=g\left(C^{\prime}\right)+g\left(C^{\prime \prime}\right)$ on exact sequences in $\mathcal{C}$ then there is a unique homomorphism $h: K^{0}(\mathrm{e}) \rightarrow G$ such that $g=h f$. Let $G_{t}(\Lambda)=K^{0}(\Im / P(\Lambda))$ and $G(\Lambda)=K^{0}(\mathfrak{T} / \odot(\Lambda))$. An immediate corollary

Received by the editors March 5, 1966 and, in revised form, July 11, 1966.

1 This research was partially supported by the National Science Foundation NSF GP 5478. 
to Theorem 1 is

COROLlaRY. The functors $F$ and $G$ induce isomorphisms

$$
\begin{aligned}
G_{t}\left(\Lambda_{1}\right) & \rightarrow G_{t}\left(\Lambda_{2}\right) \rightarrow G_{t}(\Gamma), \\
G(\Lambda)_{1} & \rightarrow G\left(\Lambda_{2}\right) \rightarrow G(\Gamma) .
\end{aligned}
$$

In case $A$ is a Dedekind domain these results are known, so in a sense Theorem 1 may be considered to be a generalization of the Morita Theorems which give these isomorphisms in this case (see [5]).

If $M$ is an $A$-lattice in $\Sigma$, define $M^{-1}=\{x \in \Sigma: M x M \subseteq M\}$. Let $\Lambda$ be a maximal order in $\Sigma$. Let $I(\Lambda)$ denote the set of $A$-lattices in $\Sigma$ which are both left and right $A$-modules. Goldman in [6] defined $D(\Lambda)$, the group of divisors of $\Lambda$, to be the abelian group obtained from $I(\Lambda)$ by the equivalence relation (quasi-equality for two-sided fractionary $\Lambda$-ideals).

$$
\text { " } M \sim N \text { in } I(\Lambda) \text { iff } \quad M^{-1}=N^{-1} . "
$$

Thus $D(\Lambda)=I(\Lambda) / \sim$, with multiplication given by $(M, N) \rightarrow \bar{M} \bar{N}$. Goldman proves that $D\left(\Lambda_{1}\right)$ is naturally isomorphic to $D\left(\Lambda_{2}\right)$ when $\Lambda_{1}$ and $\Lambda_{2}$ are maximal orders in $\Sigma$. The second result of this note is

Theorem 2. $D(\Lambda)$ is isomorphic to $G_{t}(\Lambda)$.

Theorem 2 and the corollary to Theorem 1 yield the important, but not surprising, result, namely the

Corollary. If $\Lambda$ is a maximal order in $\Sigma$, and $\Gamma$ a maximal order in $D$, then $D(\Lambda)$ is (naturally) isomorphic to $D(\Gamma)$.

Thus considerations of $D(\Lambda)$ are reduced to considerations of $D(\Gamma)$, but $\Gamma$ is in a division algebra.

1. Proof of Theorem 1. The notations of $\$ 0$ are retained here. Let $\Lambda$ and $\Omega$ be maximal $A$-orders in $\Sigma$. The conductor, $\{x \in \Sigma: \Omega x \subseteq \Lambda\}$, is denoted by $\Lambda: \Omega$. It is an $A$-lattice in $\Sigma$ which is a right ideal in $\Lambda$ and a left $\Omega$-module. Define $F(\Lambda, \Omega): \mathfrak{T}(\Lambda) \rightarrow \mathfrak{N}(\Omega)$ by $F(\Lambda, \Omega)(M)$ $=\Lambda: \Omega \otimes_{\Lambda} M$ for the left $\Lambda$-module $M$. Certainly $F(\Lambda, \Omega)$ is a functor. Since $A_{\mathfrak{p}}$ is a flat $A$-module for each prime ideal $\mathfrak{p}$ of $A$, it is clear that $A_{\mathfrak{p}} \otimes_{A} F(\Lambda, \Omega)=F\left(\Lambda_{\mathfrak{p}}, \Omega_{\mathfrak{p}}\right)$ for each prime ideal $\mathfrak{p}$ of $A$. Hence $F$ takes torsion modules to torsion modules, and pseudo-nul modules to pseudo-nul modules, and consequently induces functors

$$
\begin{aligned}
& F^{\prime}(\Lambda, \Omega): \mathfrak{T} / \odot(\Lambda) \rightarrow \mathfrak{T} / \odot(\Omega), \\
& F^{\prime \prime}(\Lambda, \Omega): \Im / \odot(\Lambda) \rightarrow J / \odot(\Omega) .
\end{aligned}
$$

( $F^{\prime \prime}$ is induced by $F^{\prime}$.) 
To show that $F^{\prime}$ (and hence $F^{\prime \prime}$ ) is an isomorphism, it is sufficient to construct a functorial inverse. But, consider the natural transformation

$$
F(\Omega, \Lambda) F(\Lambda, \Omega) \rightarrow I_{\mathscr{T T}(\Lambda)}
$$

given by $(\Omega: \Lambda) \otimes_{\Omega}(\Lambda: \Omega) \otimes_{\Lambda} M \rightarrow M: \omega \otimes \lambda \otimes m \rightarrow \omega \lambda m$. Upon localizing at a height one or less prime ideal of $A$, one obtains an identification; that is, $F\left(\Omega_{\mathfrak{p}}, \Lambda_{\mathfrak{p}}\right) F\left(\Lambda_{\mathfrak{p}}, \Omega_{\mathfrak{p}}\right)=I$. For in case $\mathfrak{p}=0, \Omega_{\mathfrak{p}}=\Sigma=\Lambda_{\mathfrak{p}}$, and in the other cases, $A_{\mathfrak{p}}$ is a discrete rank-one valuation ring, so $\Lambda_{\mathfrak{p}}: \Omega_{\mathfrak{p}}=u \Lambda_{\mathfrak{p}}=\Omega_{\mathfrak{p}} u$ and $\Omega_{\mathfrak{p}}: \Lambda_{\mathfrak{p}}=u^{-1} \Omega_{\mathfrak{p}}=\Lambda_{\mathfrak{p}} u^{-1}$, where $u$ is a unit in $\Sigma$ (by 3.4 of [1]). Hence $F^{\prime}$ (and so $F^{\prime \prime}$ ) is an isomorphism.

Using the same arguments, one shows that $F^{\prime}(\Lambda, \Omega) F^{\prime}\left(\Omega, \Omega^{\prime}\right)$ $=F^{\prime}\left(\Lambda, \Omega^{\prime}\right)$ for maximal $A$-orders in $\Sigma$. This says that the isomorphisms are natural.

Before proving the second part of Theorem 1, a generalization of Proposition 4.2 of [1] is needed.

The proof is exactly as in [1]. Proposition 4.1 of [1] and its proof remain valid when $\mathrm{Hom}$ is replaced by $\mathrm{Hom}_{\Gamma}$ and $\otimes$ by $\otimes_{\Gamma}$, so it can be used as in the proof of [1, Proposition 4.2].

Proposition 1. Let $A$ be a noetherian integrally closed integral domain with quotient field $K$. Let $\Sigma$ be a finite-dimensional central simple $K$-algebra. Suppose $\Sigma=\operatorname{Hom}_{D}(V, V)$ where $D$ is a central division $K$ algebra and $V$ a finite-dimensional right $D$-module. An A-order $\Lambda$ in $\Sigma$ is maximal if, and only if, there is a maximal $A$-order $\Gamma$ in $D$ and a right $\Gamma$-submodule $E$ of $V$ which is a reflexive $A$-lattice such that $\Lambda$ $=\operatorname{Hom}_{\Gamma}(E, E)$. In this case $\Gamma=\operatorname{Hom}_{\Lambda}(E, E)$.

Let $\Lambda$ be a maximal order in $\Sigma$ and let $E$ and $\Gamma$ be as in Proposition 1. Define $G(\Lambda, \Gamma): \mathfrak{T}(\Lambda) \rightarrow \mathfrak{T}(\Gamma)$ by $G(\Lambda, \Gamma)(M)=\operatorname{Hom}_{\Gamma}(E, \Gamma) \otimes_{\Lambda} M$. The localization arguments used above show that $G(\Lambda, \Gamma)$ preserves torsion and pseudo-nullity, so $G$ induces

$$
\begin{aligned}
& G^{\prime}(\Lambda, \Gamma): \mathfrak{T} / \odot(\Lambda) \rightarrow \mathfrak{M} / \odot(\Gamma), \\
& G^{\prime \prime}(\Lambda, \Gamma): J / P(\Lambda) \rightarrow J / \odot(\Gamma) .
\end{aligned}
$$

There is also the functor $G(\Gamma, \Lambda): \mathfrak{M}(\Gamma) \rightarrow \mathfrak{M}(\Lambda)$ defined by $G(\Gamma, \Lambda)(N)=E \otimes_{\Gamma} N$. As before, there are natural transformations

$$
\begin{aligned}
& G(\Lambda, \Gamma) G(\Gamma, \Lambda) \rightarrow \operatorname{IN}_{(\Gamma)}, \\
& G(\Gamma, \Lambda) G(\Lambda, \Gamma) \rightarrow \operatorname{IT}_{(\Lambda)} .
\end{aligned}
$$

The first is given by the natural homomorphism $\operatorname{Hom}_{\Gamma}(E, \Gamma) \otimes_{\Delta} E$ $\rightarrow \operatorname{Hom}_{\Lambda}(E, E)=\Gamma$, the second by $E \otimes_{\Gamma} \operatorname{Hom}_{\Gamma}(E, \Gamma) \rightarrow \operatorname{Hom}_{\Gamma}(E, E)$ $=\Lambda$ (cf. $[1$, Proposition A.4]). Once again, these localize to identifica- 
tions so

$$
\begin{aligned}
& G^{\prime}(\Lambda, \Gamma) G^{\prime}(\Gamma, \Lambda)=I \mathscr{T} / \mathcal{P}_{(\Gamma)} \\
& G^{\prime}(\Gamma, \Lambda) G^{\prime}(\Lambda, \Gamma)=I \mathscr{T H}_{/} \mathcal{P}_{(\Lambda)} .
\end{aligned}
$$

This concludes the proof of Theorem 1.

Heller and Reiner in [4], [5] discuss the exact sequences

$$
\begin{aligned}
& K^{1}(\Sigma) \rightarrow G_{t}(\Lambda) \rightarrow G(\Lambda) \rightarrow K^{0}(\Sigma) \rightarrow 0, \\
& K^{1}(D) \rightarrow G_{t}(\Gamma) \rightarrow G(\Gamma) \rightarrow K^{0}(D) \rightarrow 0,
\end{aligned}
$$

where $A$ is a Dedekind domain.

The corollary to Theorem 1 generalizes the discussion on pp. 351352 of [5], i.e. it implies that these are isomorphic sequences for any noetherian integrally closed integral domain $A$.

Another application of the corollary to Theorem 1 is

Proposition 2. Let $A$ be a noetherian integrally closed integral domain with quotient field $K$. Let $V$ be a finite-dimensional vector space over $K$ and let $\Sigma=\operatorname{Hom}_{K}(V, V)$. Let $\Lambda$ be a maximal order in $\Sigma$. Then

$$
\begin{aligned}
G_{\iota}(\Lambda) & =D(A) \quad(\text { divisor group of } A), \\
G(\Lambda) & =C(A) \oplus Z(C(A)=\text { class group of } A) .
\end{aligned}
$$

Proof. By the corollary to Theorem $1, G_{t}(\Lambda)=G_{t}(A)$ and $G(\Lambda)$ $=G(A)$. By Proposition 11 of $\left[3, \S 4, \mathrm{n}^{\circ} 5\right], G_{t}(A)=D(A)$. By Proposition 17 of $\left[3, \S 4, \mathrm{n}^{\circ} 8\right], G(A)=C(A) \oplus \boldsymbol{Z}$.

REMARK. Theorem 2 is a generalization of this proposition.

2. Proof of Theorem 2. The proof of the theorem is exactly the proof of Proposition 11 of $\left[3, \S 4, n^{\circ} 5\right]$ modified to the present situation.

Let $\Lambda$ be a maximal $A$-order in $\Sigma$. For each prime (two-sided) ideal $\mathfrak{P}$ of $\Lambda$ of height one let div $\mathfrak{B}$ denote its image in $D(\Lambda)$. In [7] it is proved that there is a bijection, given by $\mathfrak{B} \rightarrow \mathfrak{B} \cap A$, of the set of prime ideals of height one of $\Lambda$ to the set of prime ideals of height one of $A$. Let $P(\Lambda)$ denote the set of prime ideals of $\Lambda$.

Let $M \in J(\Lambda)$. Then if $\mathfrak{p}$ is a prime ideal of $A$, the $\Lambda_{\mathfrak{p}}$-module $M_{\mathfrak{p}}$ has finite length, denoted by $l_{\mathfrak{p}}\left(M_{\mathfrak{p}}\right)$. Since $M_{\mathfrak{p}}=0$ if $M \in \mathcal{P}(\Lambda)$, there is induced a map

$$
\chi: J / \odot(\Lambda) \rightarrow D(\Lambda)
$$

defined by $\chi(M)=\sum l_{\mathfrak{p}}\left(M_{\mathfrak{p}}\right) \operatorname{div} \mathfrak{B}, \mathfrak{p}=\mathfrak{B} \cap A, \mathfrak{B} \in P(A)$. The theorem will be proved if it can be shown that $(D(\Lambda), \chi)$ satisfies the universal mapping property defining the Grothendieck group. 
For a $\Lambda$-module $M$, let Ass $M$ denote the set of prime (two-sided) ideals $\mathfrak{P}$ of $\Lambda$ such that there is a nonzero submodule $M^{\prime}$ of $M$ with $\mathrm{Ann}_{\mathbb{A}} M^{\prime \prime}=\mathfrak{P}$ for every nonzero submodule $M^{\prime \prime}$ of $M^{\prime}$ (see [7]).

Proposition 3. Let $M$ be a finitely generated left $\Lambda$-module. Then there is a chain of submodules $M=M_{0} \supset M_{1} \supset \cdots \supset M_{r}=0, r \geqq 0$, such that $M_{i} / M_{i+1}$ is isomorphic to a module $\Lambda / \mathfrak{N}_{i}, \mathfrak{N}_{i}$ a left ideal of $\Lambda$, where Ass $\Lambda / \mathfrak{N}_{i}=\left\{\mathfrak{P}_{i}\right\}$ and $\operatorname{Ann}_{\Lambda}\left(\Lambda / \mathfrak{N}_{i}\right)=\mathfrak{B}_{i}, \mathfrak{B}_{i}$ a prime ideal of $\Lambda$.

The proof is the same as for Theorem 1 of $\left[2, \S 1, n^{\circ} 4\right]$ and is omitted.

It is clear that $\chi$ is additive on exact sequences, so Proposition 3 shows that

$$
\chi(M)=\sum_{i=0}^{r-1} \chi\left(\Lambda / \Re_{i}\right)
$$

where the $\mathfrak{\Re}_{i}$ are left ideals satisfying the conclusion of Proposition 3.

The next proposition permits a study of these modules.

Proposition 4. Let $\mathfrak{B} \in P(\Lambda), \mathfrak{p}=\mathfrak{B} \cap A$. Let $\mathfrak{m}$ be a minimal left ideal in the simple $A_{\mathfrak{p}} / \mathfrak{p} A_{\mathfrak{p}}$-algebra $\Lambda_{\mathfrak{p}} / \mathfrak{P} \Lambda_{\mathfrak{p}}$. Let $\mathfrak{n}=\mathfrak{m} \cap(\Lambda / \mathfrak{B})$. Then

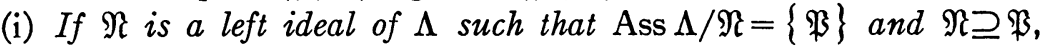
then the class of $\Lambda / \mathfrak{N}$ in $G_{t}(\Lambda)$ is some integral multiple of the class of $\mathfrak{n}$ in $G_{t}(\Lambda)$.

(ii) $\chi(\mathfrak{n})=\operatorname{div} \mathfrak{B}$.

Proof. Throughout this proof let $S=\Lambda / \mathfrak{P}$. Let $[M]$ denote the class of $M$ in $G_{t}(\Lambda)$.

Let $\mathfrak{m}_{1}$ and $\mathfrak{m}_{2}$ be two minimal left ideals in $S_{\mathfrak{p}}$. Then there is a $t$ in $S, t$ a unit in $S_{\mathfrak{p}}$, such that $\mathfrak{m}_{2}=\mathfrak{m}_{1} t$. Let $\mathfrak{n}_{i}=\mathfrak{m}_{i} \cap S$. Then $\mathfrak{n}_{1} t \subseteq \mathfrak{n}_{2}$, so consider the homomorphism $\mathfrak{n}_{1} \rightarrow^{\mathfrak{t}_{2}} \mathfrak{n}_{2}$. When localized at $\mathfrak{p}$ it is the isomorphism $\mathfrak{m}_{1} \rightarrow{ }^{t} \mathfrak{m}_{2}$. If $\mathfrak{q}$ is a prime ideal of height one of $A$ distinct from $\mathfrak{p}$, then $\left(\mathfrak{n}_{1}\right)_{\mathfrak{q}}=0=\left(\mathfrak{n}_{2}\right)_{\mathfrak{q}}$, so $t$ localized at $\mathfrak{q}$ is also an isomorphism. So in $J / \mathcal{P}(\Lambda)$ this map is an isomorphism, hence $\left[\mathfrak{n}_{1}\right]=\left[\mathfrak{n}_{2}\right]$.

Suppose that $\mathfrak{N}$ is a left ideal satisfying the hypotheses of condition (i). Then $(\mathfrak{R} / \mathfrak{B})_{\mathfrak{p}}$ is a left ideal in $S_{\mathfrak{p}}$, so is the direct sum of minimal left ideals $\mathfrak{m}_{1}, \cdots, \mathfrak{m}_{t}$ of $S_{\mathfrak{p}}$. Let $\mathfrak{n}_{i}=S \cap \mathfrak{m}_{i}$ and consider $\mathfrak{n}_{1}+\cdots+\mathfrak{n}_{t}$ in $S$. This sum is direct. The homomorphisms $\mathfrak{n}_{1}+\cdots$ $+\mathfrak{n}_{t} \rightarrow(\mathfrak{N} / \mathfrak{B})_{\mathfrak{p}} \cap S$ and $\mathfrak{N} / \mathfrak{P} \rightarrow(\mathfrak{N} / \mathfrak{B})_{\mathfrak{p}} \cap S$ are isomorphisms at every localization. Hence $t[\mathfrak{n}]=\left[\mathfrak{n}_{1}+\cdots+\mathfrak{n}_{t}\right]=[\mathfrak{N} / \mathfrak{B}]$. This holds when $\mathfrak{N}=\Lambda$, so let $[\Lambda / \mathfrak{B}]=\left[\mathfrak{n}_{1}+\cdots+\mathfrak{n}_{\mathfrak{g}}\right]=s[\mathfrak{n}]$ where $s=\left[(\Lambda / \mathfrak{P})_{\mathfrak{p}}\right.$ : $\left.(A / \mathfrak{p})_{\mathfrak{p}}\right]$. Then $t \leqq s$.

Now consider the exact sequence $0 \rightarrow \mathfrak{N} / \mathfrak{P} \rightarrow \Lambda / \mathfrak{P} \rightarrow \Lambda / \mathfrak{N} \rightarrow 0$. Then 


$$
\begin{aligned}
{[\Delta / \mathfrak{N}] } & =[\Lambda / \mathfrak{P}]-[\mathfrak{N} / \mathfrak{P}] \\
& =s[\mathfrak{n}]-t[\mathfrak{n}] \\
& =(s-t)[\mathfrak{n}] .
\end{aligned}
$$

So (i) has been established. (ii) is clear from the definition of $\mathfrak{n}$.

Corollary. For each $\mathfrak{B} \in P(\Lambda)$, let $\mathfrak{n}(\mathfrak{B})$ be a module constructed in Proposition 4. Then $G_{t}(\Lambda)$ is free on the set $[\mathfrak{n}(\mathfrak{P})]$.

This follows immediately from the two previous propositions.

Proposition 5. For each torsion left $\Lambda$-module $M$, let $g(M)$ be an element in an abelian group $G$. Suppose $g$ satisfies the two conditions

a. If $0 \rightarrow M^{\prime} \rightarrow M \rightarrow M^{\prime \prime} \rightarrow 0$ is an exact sequence in $\Im(\Lambda)$, then $g(M)$ $=g\left(M^{\prime}\right)+g\left(M^{\prime \prime}\right)$.

b. If $M \in \mathcal{P}(\Lambda)$, then $g(M)=0$.

Then there is a unique homomorphism $\theta: D(\Lambda) \rightarrow G$ such that $g=\theta \chi$.

Proof. Let $\mathfrak{n}(\mathfrak{B})$ be an ideal of $\Lambda / \mathfrak{B}$ defined in Proposition 4 . Let $\theta(\operatorname{div} \mathfrak{B})=g(\mathfrak{n}(\mathfrak{B}))$. Then continue as in Proposition 11 of $\left[3, \S 4, n^{\circ} 5\right]$. Propositions 3 and 4 are designed to make that proof work.

Proposition 5 shows that $D(\Lambda)$ satisfies the universal property which defines the Grothendieck group, so it must be isomorphic to it. This completes the proof of Theorem 2.

REMARK. Since $K^{0}(\boldsymbol{\Sigma})=K^{0}(D)=\boldsymbol{Z}$ in (HR) and $\boldsymbol{Z}$ is $\boldsymbol{Z}$ projective, $G^{0}(\Gamma)=C(\Gamma) \oplus \boldsymbol{Z}$ where $C(\Gamma)$ is the kernel of $G^{0}(\Gamma) \rightarrow K^{0}(D)$, and hence is the image of $G_{t}^{0}(\Gamma) \rightarrow G^{0}(\Gamma)$. A natural question is: What is an ideal (or module) theoretical description of the subgroup $H$ of $D(\Gamma)$ such that $D(\Gamma) / H=C(\Gamma)$ ? $C(\Gamma)$ is a generalization of the commutative class group (see [3]). A corollary to the corollary to Theorem 1 is that $C(\Lambda)$ is isomorphic to $C(\Gamma)$ and both do not depend on the maximal orders in question.

\section{BIBLIOGRAPHY}

1. M. Auslander and O. Goldman, Maximal orders, Trans. Amer. Math. Soc. 97 (1960), 1-24.

2. N. Bourbaki, Algèbre commutative, Chapitre 4, Hermann, Paris, 1961.

3. - Algèbre commutative, Chapitre 7, Hermann, Paris, 1965.

4. A. Heller and I. Reiner, Grothendieck groups of orders in semisimple algebras, Trans. Amer. Math. Soc. 112 (1964), 344-355.

5. - Grothendieck groups of integral group rings, Illinois J. Math. 9 (1965), 349-359.

6. O. Goldman, Quasi-equality in maximal orders, J. Math. Soc. Japan 13 (1961), 371-376.

7. J. A. Riley, Reflexive ideals in maximal orders, J. Algebra 2 (1965), 451-465.

The University OF IllinoIS 